\title{
Alternating Hemiplegia of Childhood: Genotype-Phenotype Correlations in a Cohort of 39 Italian Patients
}

\author{
Ramona Cordani ${ }^{1}$, Michela Stagnaro ${ }^{2}$, Livia Pisciotta ${ }^{1,3}$, Francesco Danilo Tiziano ${ }^{4}$, \\ Maria Grazia Calevo ${ }^{5}$, Lino Nobili ${ }^{1,2}$, I.B.AHC Consortium and Elisa De Grandis ${ }^{1,2 *}$ \\ ${ }^{1}$ Department of Neurosciences, Rehabilitation, Ophthalmology, Genetics, Maternal and Child Health, University of Genoa, \\ Genoa, Italy, ${ }^{2}$ Child Neuropsychiatry Unit, Department of Clinical and Surgical Neurosciences and Rehabilitation, Istituto di \\ Ricovero e Cura a Carattere Scientifico Giannina Gaslini, Genova, Italy, ${ }^{3}$ Child Neuropsychiatry Unit, Azienda Socio Sanitaria \\ Territoriale Fatebenefratelli- Sacco, Milano, Italy, ${ }^{4}$ Section of Genomic Medicine, Department of Life Science and Public \\ Health, Catholic University of Sacred Heart, Roma, Italy, ${ }^{5}$ Epidemiology, Biostatistics and Committees Unit, Istituto di \\ Ricovero e Cura a Carattere Scientifico Istituto Giannina Gaslini, Genoa, Italy
}

OPEN ACCESS

Edited by:

Anna De Rosa,

Università di Napoli Federico II, Italy

Reviewed by:

Hirokazu Oguni,

TMG Asaka Medical Center, Japan

Kishore Raj Kumar,

Garvan Institute of Medical

Research, Australia

*Correspondence:

Elisa De Grandis

elisadegrandis@gaslini.org

Specialty section:

This article was submitted to

Movement Disorders,

a section of the journal

Frontiers in Neurology

Received: 25 January 2021 Accepted: 25 February 2021

Published: 08 April 2021

Citation:

Cordani $R$, Stagnaro $M$, Pisciotta L,

Tiziano FD, Calevo MG, Nobili L,

I.B.AHC Consortium and De Grandis E

(2021) Alternating Hemiplegia of

Childhood: Genotype-Phenotype

Correlations in a Cohort of 39 Italian

Patients. Front. Neurol. 12:658451.

doi: 10.3389/fneur.2021.658451
Alternating hemiplegia of childhood is a rare neurological disease characterized by paroxysmal movement disorders and chronic neurological disturbances, with onset before 18 months of age. Mutations in the ATP1A3 gene have been identified in up to $80 \%$ of patients. Thirty-nine patients [20 females, 19 males, mean age 25.32 years (7.52-49.34)] have been recruited through the Italian Biobank and Clinical Registry for Alternating Hemiplegia of Childhood. Demographic data, genotype, paroxysmal movement disorders, chronic neurological features, and response to flunarizine have been analyzed. ATP1A3 gene mutations have been detected in 92.3\% of patients. Patients have been divided into three groups - p.Asp801Asn mutation patients (26\%), p.Glu815Lys cases (23\%), and patients with other ATP1A3 mutations - and statistically compared. The Italian cohort has a higher percentage of ATP1A3 gene mutation than reported in literature (92.3\%). Our data confirm a more severe phenotype in patients with p.Glu815Lys mutation, with an earlier age of onset of plegic $(p=0.02$ in the correlation with other mutations) and tonic attacks. P.Glu815Lys patients most frequently present altered muscle tone, inability to walk $(\rho=0.01$ comparing p.Glu815Lys and p.Asp801Asn mutations), epilepsy, and a more severe grade of dystonia ( $p<0.05$ comparing p.Glu815Lys and p.Asp801Asn mutations). They have moderate/severe intellectual disability and severe language impairment $(p<0.05)$. Interestingly, flunarizine seems to be more efficacious in patients with p.Glu815Lys mutation than p.Asp801Asn. In conclusion, our research suggests a genotype-phenotype correlation and provides information on this disorder's features, clinical course, and treatment.

Keywords: alternating hemiplegia of childhood, ATP1A3, movement disorder, genotype, phenotype, flunarizine, epilepsy

\section{INTRODUCTION}

Alternating Hemiplegia of Childhood (AHC) (OMIM \#614820) is a rare and peculiar neurologic disorder, first described in 1971 (1). The annual incidence is $<1 / 100,000$ newborns (2). In AHC, a complex phenotype combines paroxysmal non-epileptic episodes, often triggered by contact with water, changes in temperature, physical or psychological stress, and epileptic seizures, 
both focal and generalized, with a high rate of refractory/super refractory status epilepticus (3). Developmental issues, cognitive deficits, neuropsychological impairments, and persistent neurologic disorders $(2,4-6)$ are expected hallmarks. In 1993, specific diagnostic criteria for AHC were first introduced (7) and then periodically updated. Aicardi's criteria for AHC (8) are (1) onset of paroxysmal events before 18 months of age; (2) repeated bouts of hemiplegia involving the right and left sides of the body during some attacks; (3) episodes of bilateral hemiplegia or quadriplegia starting either as a generalization of a hemiplegic episode or as bilateral from the start; (4) other paroxysmal disturbances including tonic/dystonic attacks, nystagmus, strabismus, dyspnea, and other autonomic phenomena occurring during hemiplegic bouts or in isolation; (5) immediate disappearance of all symptoms upon sleep, with probable recurrence of long-lasting bouts, $10-20 \mathrm{~min}$ after awakening; (6) evidence of developmental delay, intellectual disability, neurological abnormalities, choreoathetosis, and dystonia or ataxia; and (7) not attributable to other disorders. A great effort was made to understand the disease's genetic basis. In 2012, In 2012, two independent research groups - a group of German researchers (9) and an international consortium (10)-identified de novo heterozygous mutations in the ATP1A3 gene performing next-generation sequencing to examine the genome of AHC patients (11). Next, this finding was replicated by an independent Japanese study that found ATP1A3 mutations in patients with AHC (12). Currently, pathogenic variants in the ATP1A3 gene are recognized as causing AHC in nearly $80 \%$ of patients (13). ATP1A3 gene encodes for the alpha3 subunit of $\mathrm{Na}+/ \mathrm{K}+\mathrm{ATPase}$, which is neuron-specific. Interestingly, the cerebellum seems to be highly susceptive to ATP1A3 dysfunction (14). Despite the growing number of pathogenic variants described, the most extensive cohort studies conducted in various populations showed that three variants account for $\sim 60 \%$ of all cases. The p.Asp801Asn variant is detected in $30-43 \%$ of all cases, p.Glu815Lys is responsible for $16-35 \%$ of cases, and p.Gly947Arg is responsible for $8-15 \%(13,15,16)$. Mutations in the ATP1A3 gene are associated with other neurological diseases such as rapid-onset dystonia-parkinsonism (DYT12, OMIM 128235) (9), cerebellar ataxia, areflexia, pes cavus, optic atrophy, sensorineural hearing loss (CAPOS) syndrome (OMIM \# 601338) (17), the early infantile epilepsy with encephalopathy (EIEE) (18), and the recurrent encephalopathy with cerebellar ataxia (RECA) phenotype (19). Recently, mutations of the RHOBTB2 gene have been identified as responsible for a complex movement disorder with paroxysmal elements similar to AHC, suggesting to evaluate this gene in the diagnostic workup of patients with AHC, in particular those negative for ATPA1A3 mutations (20). Although a second major causative gene has not yet been identified, other gene mutations (ATP1A2, CACNA1A, SLC1A3, SLC2A1) have occasionally been related to AHC (21-24). Concerning pharmacological approaches, the main therapy goals are prophylaxis of paroxysmal attacks and chronic disturbance treatment, as dystonia and epilepsy. Disease-modifying therapy does not exist, albeit several drugs are administered as prophylaxis for paroxysmal attacks. The most frequent drugs used in AHC are flunarizine, benzodiazepines, carbamazepine, barbiturates, and valproic acid (25). It has been widely demonstrated that flunarizine induces a significant improvement in dystonic and plegic episodes (26).

In recent years, scientific efforts are focusing on defining a genotype-phenotype correlation to better understand the pathogenesis of the disease and provide indications for management and treatment. In literature, high interindividual variability as regards the clinical evolution has been reported. However, the investigation of adult patients' outcome excludes a progressive and neurodegenerative disorder (27). This study aims to describe an Italian cohort of AHC patients' genetic and clinical features and further establishes genotype-phenotype correlation. A significant number of patients enrolled for this study had already been included in previous multicenter studies published by Heinzen and colleagues in 2012 (10) and Panagiotakaki et al. in 2015 (13). However, in this research, we examined the clinical phenotype more in detail and flunarizine efficacy in different genotypes.

\section{METHODS}

\section{Sample Patients}

Thirty-nine patients have been recruited through the I.B.AHC Italian Biobank and Clinical Registry for AHC (I.B.AHC). The diagnosis was based on Aicardi's criteria. All the patients have been diagnosed by consensus of the Scientific Association Committee of the Italian Association of Alternating Hemiplegia of Childhood (AISEA). Patients' referring pediatric neurologists have filled patients' forms, and I.B.AHC data managers validated the information's accuracy.

\section{Mutation Analysis}

DNA was obtained from the blood of the probands and parents using standard procedures. ATP1A3 mutations were identified by Sanger (34/39 patients), whole-exome (3/39), or next-generation sequencing for a targeted panel (2/39) for ATP1A3 and other AHC-related genes. The mutation analysis was extended, whenever possible, to the parents to define if the mutation occurred de novo. Variant nomenclature was attributed based on RefSeq (NG_008015.1 and NM_152296, for genomic and mRNA sequences, respectively). Pathogenicity was revised following the original description, according to the ACMG criteria (28).

\section{Data Collection}

Clinical, demographic, genetic, and pharmacological data of the 39 Italian patients have been collected. In more detail, data concerning paroxysmal movement disorders, particularly age of onset, type of episode as hemiplegic and tonic attacks, ocular abnormalities and autonomic dysfunctions, and the effect of sleep on episodes were examined. Chronic neurological features at last follow-up, especially muscle tone, gait characteristics, movement disorders (dystonia, chorea, myoclonus, postural or action tremor, bradykinesia), migraine, and the presence of intellectual disability and of varying degrees of language impairment, were also collected. The occurrence of reduced tone or hypertonia due to spasticity was defined "altered muscle tone." 
For each patient, gait was assessed, and a differentiation was made between cases with normal gait, wide-based gait, and walking with trunk dystonia ("gait possible"), and subjects able to walk ("gait not possible"). Three degrees of dystonia severity have been identified by differentiating in mild dystonia when not interfering with function, moderate dystonia when interfering with activities in daily life, and severe dystonia when disabling. Intellectual disability was classified as "mild" (IQ of 50-69), "moderate" (IQ of 35-49), or "severe" (IQ < 35). Language impairment was stated as mild when not affecting communication abilities, moderate when partially affecting communication abilities, or severe when affecting communication abilities. A distinction was made between AHC typical form (according to Aicardi's criteria for AHC) and atypical forms (24). This last definition was used in case of delayed occurrence of hemiplegic attacks, normal development, predominant dystonic phenotype, absence of quadriplegic attacks, or alternating upper-limb monoplegia. Lastly, the response to treatment with flunarizine was analyzed, considering as an improvement a reduction of at least 30\% in duration, frequency, and intensity of the episodes.

\section{Statistical Analysis}

Data are described as mean and standard deviation or median and range for continuous variables and as absolute and relative frequencies for categorical variables. Nonparametric analysis (Mann-Whitney $U$-test) for continuous variables and the Chi square or Fisher's exact test for categorical variables were used to measure differences between groups. Statistical significance was set as 0.017 using Bonferroni correction; all $p$-values were based on two-tailed tests. Statistical analysis was performed using SPSS for Windows (SPSS Inc., Chicago, Illinois USA). By meaning of ATP1A3 mutation, patients were divided into three groups: patients with p.Asp801Asn mutation, patients with p.Glu815Lys mutation, and patients with other mutations, and statistically compared.

\section{RESULTS}

\section{Genetic Findings}

ATP1A3 gene mutations have been detected in $92.3 \%$ of patients (36/39): $23 \%(9 / 39)$ of patients had a p.Glu815Lys mutation, $26 \%(10 / 39)$ p.Asp801Asn mutation, and 43.6\% (17/39) other various mutations. The more frequent gene mutation in the latter group was p.Gly947Arg (4/17; 10\% of total patients). After revision of pathogenicity according to the ACMG guidelines (28), all variants were interpreted as Pathogenic (Class 5) since responding to the following criteria: PS1, PS3, PM2, PM6, PP2, PP3, and PP4.

Three out of 39 patients had no ATP1A3 mutations. Tables 1, 2 summarize demographic data and paroxysmal and nonparoxysmal features in different group mutations.

\section{Demographic Data}

The average age of the whole group at the time of data collection was $18 \pm 10.68$ years (Table 1). In particular, patients with p.Glu815Lys mutation had $25.1 \pm 10$ years at the time of data collection, patients with p.Asp801Asn mutation $32.74 \pm$
10.6 years, and those with other mutations $20.6 \pm 10.2$ years. Comparing the different groups, the recruited patients with p.Asp801Asn mutation were significantly older than those with various mutations $(p=0.007)$. No differences concerning patient gender have been observed (20 female and 19 males). However, analyzing specific groups, a female prevalence in patients with p.Glu815Lys (67\%) and p.Asp801Asn mutation (70\%) has been detected. Vice versa, a male prevalence has been observed in the group with various mutations $(71 \%)$, albeit without statistically significant differences $(p=0.06$ comparing with p.Asp801Asn and $p=0.10$ with p.Glu815Lys patients). All patients with p.Asp801Asn mutation had a typical AHC. Atypical AHC forms were more frequent in p.Glu815Lys (44 vs. $0 \%$ in cases with p.Asp801Asn- $p=0.03$ ) and $23.5 \%$ in cases with other mutations $(p=0.26)$.

\section{Paroxysmal Features}

Concerning paroxysmal events (Table 1), the onset of plegic episodes occurred at $5.88 \pm 0.83$ months in patients with p.Glu815Lys mutation, $6.8 \pm 2.7$ months in p.Asp801Asn patients, and $13.4 \pm 9.1$ months in patients with other mutations. The age of onset of plegic episodes was lower in p.Glu815Lysmutated cases compared to the group with various mutations $(p=0.02)$. Tonic attacks appeared at $3.2 \pm 2.3$ months in p.Glu815Lys patients, at $11.1 \pm 25.8$ months in patients with p.Asp801Asn mutation, and $7.8 \pm 8.1$ months in patients with other mutations. Abnormal eye movements began at $2.6 \pm 2.5$ months in patients with p.Glu815Lys mutation, $2.4 \pm 2.8$ months in p.Asp801Asn patients, and $14.2 \pm 38.2$ months in patients with other mutations. No statistically significant differences were detected between the three groups as far as age of onset of paroxysmal attacks, albeit patients with p.Glu815Lys mutation globally presented an earlier onset than the other groups with a $p$-value approaching statistical significance. All patients with p.Glu815Lys and p.Asp801Asn mutations and 94\% (16/17) with other mutations had hemilateral plegic attacks at follow-up. Moreover, from 60 to $67 \%$ of the mutated patients had total plegic attacks. All patients with p.Asp801Asn mutation, 89\% (8/9) with p.Glu815Lys, and 75\% (12/16) with other mutations had tonic attacks. Autonomic dysfunctions have been observed in $90 \%(9 / 10)$ of patients with p.Asp801Asn mutation, in $77.8 \%$ (7/9) of p.Glu815Lys patients, and in $64.7 \%$ (11/17) of other mutations. Lastly, abnormal eye movements have been detected in all p.Glu815Lys patients, in $80 \%(8 / 10)$ of patients with the p.Asp801Asn mutation, and $88 \%(15 / 17)$ of patients with other mutations. Symptoms were relieved by sleep in 78 to $94 \%$ of cases (the higher percentage in patients with other mutations, the lower in p.Glu815Lys mutation). Paroxysmal plegic and tonic attacks, autonomic dysfunctions, and paroxysmal eye movements have been widespread throughout the analyzed sample without statistically significant differences in the different groups.

\section{Non-paroxysmal Movement Disorders}

Muscle tone was assessed by neurological examination. Statistical analysis compared patients with normal muscle tone to subjects with altered muscle tone (including both decreased and increased tone) in the three groups without finding any statistically 
TABLE 1 | Demographic data and paroxysmal episode features in the three mutational groups.

\begin{tabular}{|c|c|c|c|c|c|c|}
\hline & $\begin{array}{l}\text { Group 1: } \\
\text { Various } \\
\text { mutations } \\
N=17\end{array}$ & $\begin{array}{l}\text { Group 2: } \\
\text { p.Asp801Asn } \\
\text { mutation } \\
N=10\end{array}$ & $\begin{array}{l}\text { Group 3: } \\
\text { p.Glu815Lys } \\
\text { mutation } \\
N=9\end{array}$ & $p=1$ vs. 2 & $p=1$ vs. 3 & $p=2$ vs. 3 \\
\hline \multicolumn{7}{|l|}{ Age, years } \\
\hline Mean \pm SD & $20.6 \pm 10.2$ & $32.7 \pm 10.6$ & $25.1 \pm 10$ & & & \\
\hline \multirow[t]{2}{*}{ Median (range) } & 18.5 (7.5-49.3) & $32.2(11.1-47.5)$ & 22.4 (12.9-39.5) & 0.007 & 0.26 & 0.13 \\
\hline & $N(\%)$ & $N(\%)$ & $N(\%)$ & & & \\
\hline Gender (M) & $12(70.6)$ & $3(30)$ & 3 (33.3) & 0.06 & 0.10 & 1 \\
\hline Typical AHC & $13(76.5)$ & $10(100)$ & 5 (55.6) & 0.26 & 0.38 & 0.03 \\
\hline PA onset, months & $13.4 \pm 9.1$ & $6.8 \pm 2.7$ & $5.88 \pm 0.83$ & 0.07 & 0.02 & 0.26 \\
\hline TA onset, months & $7.8 \pm 8.1$ & $11.1 \pm 25.8$ & $3.2 \pm 2.3$ & 0.40 & 0.12 & 0.84 \\
\hline EA onset, months & $14.2 \pm 38.2$ & $2.4 \pm 2.8$ & $2.6 \pm 2.5$ & 0.42 & 0.37 & 0.96 \\
\hline Hemilateral PA & $16(94.1)$ & $10(100)$ & $9(100)$ & 1 & 1 & - \\
\hline Total PA & $11(64.7)$ & $6(60)$ & $6(66.7)$ & 1 & 1 & 1 \\
\hline Mixed PA & $7(43.8)$ & $7(70)$ & $6(66.7)$ & 0.24 & 0.41 & 1 \\
\hline Tonic attacks & $12(75)$ & $10(100)$ & 8 (88.9) & 0.14 & 0.62 & 0.47 \\
\hline Hemilateral TA & 7 (58.3) & $7(70)$ & $4(44.4)$ & 0.67 & 0.67 & 0.37 \\
\hline Total TA & 9 (69.2) & $7(70)$ & $6(66.7)$ & 1 & 1 & 1 \\
\hline Autonomic dysfunction & $11(64.7)$ & $9(90)$ & 7 (77.8) & 0.20 & 0.67 & 0.58 \\
\hline $\begin{array}{l}\text { Paroxysmal eye } \\
\text { abnormalities }\end{array}$ & $15(88.2)$ & $8(80)$ & $9(100)$ & 0.61 & 0.53 & 0.47 \\
\hline Sleep effect & $16(94.1)$ & $8(80)$ & $7(77.8)$ & 0.53 & 0.27 & 1 \\
\hline
\end{tabular}

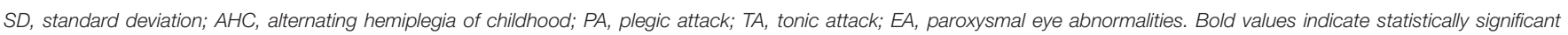
$p$ values.

significant difference (Table 2). Globally, reduced or increased muscle tone has been detected in $85 \%$ of the whole cohort (33/39, including 25 patients with hypotonia and eight with spastic hypertonia). Analyzing specific groups, all patients with p.Glu815Lys mutation (6/9 presented hypotonia and 3/9 hypertonia), 70\% (7/10, 6 with hypotonia and 1 with hypertonia) with p.Asp801Asn, and $82.4 \%$ (14/17, 11 with reduced muscle tone and three with hypertonia) with other mutations presented abnormal muscle tone. At the last follow-up, $77 \%$ of patients walked independently. Twenty-eight percentage of them had wide-based gait, and $10 \%$ had trunk dystonia. Walking was not possible in $56 \%(5 / 9)$ of cases with p.Glu815Lys mutation and $17.6 \%(3 / 17)$ of patients with other mutations. Vice versa, gait was possible in all patients with the p.Asp801Asn mutation, although in some cases with dystonia and wide-based gait. The comparison of this latter group with the p.Glu815Lys mutated patients showed statistically significant values $(p=$ 0.01). AHC Italian patients present a wide range of movement disorders, although they were not significantly different between the three groups (Table 2). Dystonia was observed in $85 \%$ of patients, most of whom had generalized dystonia ( $80 \%$ of cases). Patients with the highest percentage of dystonia were those with p.Asp801Asn mutation (90\%, 9/10); lower percentages were found in p.Glu815Lys patients $(78 \%, 7 / 9)$ and patients with other mutations $(82 \%, 14 / 17)$. Statistical analysis compared cases with absent or mild dystonia with subjects with moderate or severe dystonia. Seventy-five percentage (6/8) of patients with the p.Glu815Lys mutation had moderate/severe dystonia, whereas all patients with p.Asp801Asn had a mild form; the difference in dystonia severity between p.Glu815Lys and p.Asp801Asn cases approached statistical significance $(p=0.002)$. On the other hand, dystonia in patients with various mutations was absent or mild in $64.7 \%(11 / 17)$ and moderate/severe in $35.3 \%(6 / 17)$ of cases. Myoclonus was noticed in $31 \%$ of patients ranging from $22 \%$ in p.Glu815Lys mutation to $35 \%$ in other mutations. Action and postural tremor were observed in $15.4 \%$ of cases: the higher percentage in p.Glu815Lys mutation (33\%) and the lower percentage in p.Asp801Asn (10\%).Chorea has been detected in $13 \%$ of mutated patients $(17.6 \%$ of patients with various mutations, $10 \%$ of p.Asp 801 Asn cases, and $14 \%$ of patients with p.Glu815Lys mutation). Finally, bradykinesia has been detected only in the group of patients with p.Asp801Asn mutation (20, $5.4 \%$ of all patients).

\section{Epilepsy}

As far as epilepsy is concerned (Table 2), 62\% (24/39) of patients experienced epileptic seizures, both focal (37\%) and generalized (26\%). By meaning ATP1A3 mutation, epilepsy was more frequent in cases with p.Glu815Lys mutation $(89 \%, 8 / 9)$, in respect to $60 \%(6 / 10)$ with p.Asp801Asn and about 53\% $(9 / 17)$ with other mutations ( $p=0.09$ compared to the p.Glu815Lys mutation group). Besides, half of the patients with p.Glu815Lys mutation had a status epilepticus.

\section{Migraine}

Forty-four percentage of all patients suffered from headache, with a higher percentage in the p.Asp801Asn mutation group (56\%, 
TABLE 2 | Epilepsy, non-paroxysmal movement disorders, and cognition in the three mutational groups.

\begin{tabular}{|c|c|c|c|c|c|c|}
\hline & $\begin{array}{l}\text { Group 1: } \\
\text { various } \\
\text { mutations } \\
N=17\end{array}$ & $\begin{array}{l}\text { Group 2: } \\
\text { p.Asp801Asn } \\
\text { mutation } \\
N=10\end{array}$ & $\begin{array}{l}\text { Group 3: } \\
\text { p.Glu815Lys } \\
\text { mutation } \\
N=9\end{array}$ & $p=1$ vs. 2 & $p=1$ vs. 3 & $p=2$ vs. 3 \\
\hline & $N(\%)$ & $N(\%)$ & $N(\%)$ & & & \\
\hline Epilepsy & $9(52.9)$ & $6(60)$ & 8 (88.9) & 1 & 0.09 & 0.30 \\
\hline Status epilepticus & $2(11.8)$ & $2(20)$ & $4(50)$ & 0.61 & 0.06 & 0.32 \\
\hline \multicolumn{7}{|l|}{ Muscle tone } \\
\hline Normal & $3(17.6)$ & $3(30)$ & - & 0.64 & 0.53 & 0.21 \\
\hline Reduced/increased & $14(82.4)$ & $7(70)$ & $9(100)$ & & & \\
\hline Ataxia & - & - & $1(11.1)$ & & 0.35 & 0.47 \\
\hline \multicolumn{7}{|l|}{ Gait } \\
\hline $\begin{array}{l}\text { Possible } \\
\text { (normal/wide-based/trunk } \\
\text { dystonia) }\end{array}$ & $14(82.4)$ & $10(100)$ & $4(44.4)$ & 0.27 & 0.08 & 0.01 \\
\hline Not possible & $3(17.6)$ & - & $5(55.6)$ & & & \\
\hline Dystonia & $14(82.4)$ & $9(90)$ & $7(77.8)$ & 1 & 1 & 0.58 \\
\hline \multicolumn{7}{|l|}{ Dystonia severity } \\
\hline No/mild & $11(64.7)$ & $9(100)$ & $2(25)$ & 0.06 & 0.09 & 0.002 \\
\hline Moderate/severe & $6(35.3)$ & - & $6(75)$ & & & \\
\hline Myoclonus & $6(35.3)$ & $3(30)$ & $2(22.2)$ & 1 & 0.67 & 1 \\
\hline Action/postural tremor & $2(11.8)$ & $1(10)$ & $3(33.3)$ & 1 & 0.30 & 0.30 \\
\hline Chorea & $3(17.6)$ & $1(10)$ & $1(13.9)$ & 1 & 1 & 1 \\
\hline Bradykinesia & - & $2(20)$ & - & 0.14 & - & 0.47 \\
\hline Migraine & $8(47.1)$ & $5(55.6)$ & 3 (33.3) & 1 & 0.68 & 0.64 \\
\hline \multicolumn{7}{|l|}{ Intellectual disability } \\
\hline No/mild & $8(47.1)$ & $4(40)$ & - & 1 & 0.02 & 0.09 \\
\hline Moderate/severe & $9(52.9)$ & $5(50)$ & $9(100)$ & & & \\
\hline \multicolumn{7}{|l|}{ Speech impairment } \\
\hline No/mild & $9(52.9)$ & $7(70)$ & - & 0.45 & 0.009 & 0.003 \\
\hline Moderate/severe & $8(47.1)$ & $3(30)$ & $9(100)$ & & & \\
\hline
\end{tabular}

Bold values indicate statistically significant $p$ values.

5/9-information not available for one patient), followed by the group with various mutations $(47 \%, 8 / 17)$ (Table 2$)$.

\section{Language and Cognitive Features}

Regarding cognitive features (Table 2), a statistical comparison was made between the group with mild impairment and the group with moderate or severe intellectual disability. Moderate to severe impairment was observed in all patients with the p.Glu815Lys mutation. Lower percentages were found in other patients: $53 \%(9 / 17)$ of patients with other mutations $(p=0.02)$ and half of those with the p.Asp801Asn mutation $(5 / 10)(p=0.09)$. Furthermore, all p.Glu815Lys patients, $47 \%(8 / 17)$ with other mutations $(p=0.009)$ and $30 \%(3 / 10)$ of p.Asp801Asn patients $(p=0.003)$, exhibited moderate/severe speech impairment (absent or significantly reduced expressive language).

\section{Flunarizine Treatment Effect}

Table 3 summarizes flunarizine effects in the three different mutational groups. Thirty-four patients received flunarizine (thirty-one patients with ATP1A3 gene mutation and three patients without mutation). In patients with p.Glu815Lys mutation, this drug reduced the intensity and duration of episodes $(>30 \%)$ in $67 \%(6 / 9)$ of cases and produced a decrease of frequency in $89 \%$ (8/9). Thirty-three of patients with p.Asp801Asn mutation (3/9) presented reduced intensity and frequency; $44 \%$ shorter duration. The $77 \%$ (10/13) of patients with other mutations had a reduction in episode intensity and the $85 \%(11 / 13)$ reduction in duration and frequency. Compared with the other two groups, patients with p.Asp801Asn mutations seemed to present a minor response to flunarizine; in particular, the episode frequency reduction was considerably lower than in other groups. In none of the patients examined, flunarizine produced a worsening of the clinical features.

\section{Patients Without ATP1A3 Gene Mutation}

The group of patients with no gene mutation exhibits somewhat heterogeneous clinical features. All patients had plegic attacks that developed from 0 to 24 months of age, abnormal paroxysmal eye movements, and total tonic attacks. In two out of three patients, paroxysmal events were relieved by sleep. One patient had epilepsy and status epilepticus, and all had dystonia, from mild to severe degree. In one patient, walking was not possible, and two patients showed 
TABLE 3 | Flunarizine effect in the three mutational groups.

\begin{tabular}{|c|c|c|c|c|c|c|}
\hline Flunarizine effect & $\begin{array}{l}\text { Group 1: } \\
\text { Various } \\
\text { mutations } \\
N=13\end{array}$ & $\begin{array}{l}\text { Group 2: } \\
\text { p.Asp801Asn } \\
\text { mutation } \\
N=9\end{array}$ & $\begin{array}{l}\text { Group 3: } \\
\text { p.Glu815Lys } \\
\text { mutation } \\
N=9\end{array}$ & $p=1$ vs. 2 & $p=1$ vs. 3 & $p=2$ vs. 3 \\
\hline \multicolumn{7}{|l|}{ Episode intensity } \\
\hline Reduction $\geq 30 \%$ & $10(76.9)$ & $3(33.3)$ & $6(66.7)$ & 0.07 & 0.65 & 0.35 \\
\hline Reduction $<30 \% /$ no effect & $3(23.1)$ & $6(66.7)$ & 3 (33.3) & & & \\
\hline \multicolumn{7}{|l|}{ Episode duration } \\
\hline Reduction $\geq 30 \%$ & $11(84.6)$ & $4(44.4)$ & $6(66.7)$ & 0.07 & 0.61 & 0.64 \\
\hline Reduction $<30 \% /$ no effect & $2(15.4)$ & $5(55.6)$ & 3 (33.3) & & & \\
\hline \multicolumn{7}{|l|}{ Episode frequency } \\
\hline Reduction $\geq 30 \%$ & $11(84.6)$ & 3 (33.3) & 8 (88.9) & 0.03 & 1 & 0.05 \\
\hline Reduction $<30 \% /$ no effect & $2(15.4)$ & $6(66.7)$ & $1(11.1)$ & & & \\
\hline
\end{tabular}

The flunarizine effect on paroxysmal attacks was evaluated considering an improvement of at least 30\% in the episodes' duration, frequency, and intensity.

a wide-based gait. They all had an intellectual disability (one mild and two moderate) and speech impairment (absent or significantly reduced expressive language). Finally, in one out of three patients, flunarizine resulted in a reduction of more than $30 \%$ in frequency, duration, and intensity of the episodes.

\section{DISCUSSION}

Our study reports genotype-phenotype correlation and provides information on paroxysmal and non-paroxysmal features, clinical course, and treatment of a cohort of 39 Italian AHC patients. The Italian patients have a higher percentage of ATP1A3 gene mutation (92.3\%) than as described in literature (13). The three most frequent mutations are p.Asp801Asn, p.Glu815Lys, and p.Gly947Arg, confirming findings already reported in the literature (15). Although an increasing number of pathogenic variants have been described, the variants listed above have been found to cause about $60 \%$ of all cases in the most extensive cohort studies. In particular, the p.Asp801Asn variant accounts for $30-43 \%$ of all cases, p.Glu815Lys 16$35 \%$ of cases, and p.Gly947Arg 8-15\% (25). No other genes occasionally related to AHC patients (21-24) were detected in this case series. Previous studies showed a more severe phenotype in p.Glu815Lys mutation, with an earlier onset of symptoms, drug-resistant epilepsy, dystonia, and severe intellectual disability as main neurological findings $(11,13)$. Interestingly, a mouse model of AHC (ATP1A3 E815K+/-, Matoub, Matb ${ }^{+-}$) expressing the E815K mutation of the ATP1A3 gene has recently been reported. In this study, the authors demonstrated that mutated mice exhibit features comparable to AHC patients with the same mutation. Mutated mice have poor motor initiative and profoundly impaired motor performance, particularly concerning coordination and abnormal gait. Of note, the hemiplegia and dystonia episodes were both spontaneous and induced by a high level of stress, similar to the human phenotype. Besides, the mouse model presented spontaneous seizures or induced seizures and sudden unexpected death in epilepsy (SUDEP). Although there are differences between the proposed models, some features are common: brain hyperexcitability, motor abnormalities (spontaneous or provoked), and behavioral alterations, according to a fundamental role of ATP1A3 in brain functioning (29). In our sample, patients with p.Glu815Lys mutation most frequently present altered muscle tone, inability to walk $(p=0.01$ comparing p.Glu815Lys and p.Asp801Asn mutations), epilepsy, and a more severe grade of dystonia $(p=0.002$ comparing p.Glu815Lys and p.Asp801Asn mutations), confirming a worse phenotype related to this mutation. Moreover, these patients have moderate/severe intellectual disability and moderate/severe language impairment compared to other groups. In particular, the language appears more injured in this group, reaching significant values in statistical analysis $(p=0.009$ and $p=0.003)$. Regarding paroxysmal episodes, our data show that p.Glu815Lys patients present an earlier age of onset of plegic $(p=0.02$ in the correlation with other mutations) and tonic attacks. Furthermore, four out of nine of our p.Glu815Lys patients presented rapid motor decline and language loss and a sudden worsening of dystonia. As far as psychomotor deterioration is concerned, we assume that a genetic determinant could be involved considering that severe deterioration with no recovery is typical of Rapid Onset Dystonia Parkinsonism, another allelic disorder caused by the ATP1A3 mutation. Panagiotakaki and colleagues (13) after reviewing clinical data of a large cohort of 155 patients from the International Consortium of AHC reported a milder phenotype in p.Asp801Asn patients with later onset of the paroxysmal events, moderate intellectual disability, and a higher behavioral problems rate. Similarly, in our study, patients with this mutation had milder clinical signs than the p.Glu815Lys group. All patients had independent walking, and a less percentage of cases had alterations in muscle tone on neurological examination. Besides, ataxia, chorea, and action and postural tremor were less frequent than in other groups. However, a higher prevalence of dystonia was observed than in other groups ( $90 \%$ of cases), although no patients had moderate/severe forms (common finding in patients with the 
p.Glu815Lys mutation, $p=0.002)$. As for the cognitive aspects, they had a lower percentage of moderate/severe intellectual disability. The group with various mutations exhibited a later onset of paroxysmal hemiplegic attacks and abnormal eye movements. Concerning non-paroxysmal neurologic features, a more severe neurological impairment than patients with p.Asp801Asn mutation has been detected. Interestingly, in this group, a male prevalence was observed differently from other groups. It is well known that AHC patients harboring ATP1A3 mutations exhibit various forms of hyperkinetic non-paroxysmal movement disorders as chorea, dystonia, myoclonus, and ataxia, with a heterogeneous degree of severity. Our study does not confirm previous study results. In fact, in French patients, choreoathetosis and ataxia have been described in all cases (7), and in US patients, ataxia was reported in $68 \%$ of cases (8). In a recent research, a lower rate of non-paroxysmal movement disorders has been reported (30). It is reasonable to hypothesize that these differences between studies are partly due to the complexity in assessing movement disorders' phenomenology. Further, this is the first study aiming to analyze flunarizine's effect on paroxysmal episodes in different mutational groups. Flunarizine, a calcium antagonist, reduces the duration, severity, and frequency of paroxysmal attacks in up to $80 \%$ of cases in some studies $(8,26)$. The exact mechanism by which flunarizine improves paroxysmal spells in AHC is not yet fully recognized. Flunarizine may be useful in patients with $\mathrm{Na}+/ \mathrm{K}+$ pump dysfunction by blocking voltage-gated $\mathrm{Na}+$ and $\mathrm{Ca}+$ currents, limiting neuronal hyperexcitability, and increasing the threshold to develop cortical spreading depression (29). Our study confirms the benefit of flunarizine on paroxysmal episodes and highlights differences of effects on different mutations. Comparing the two most frequent ATP1A3 mutations, flunarizine seems to be more efficacious in patients with p.Glu815Lys mutation than p.Asp801Asn, in reducing intensity, frequency, and duration of paroxysmal episodes. Interestingly, Helseth et al. demonstrated that flunarizine also reduced hemiplegic attacks and seizures in E815K (p.Glu815Lys) mouse models (29). A positive response to flunarizine treatment was also observed in the group of patients with various mutations. Greater efficacy of reducing the intensity, duration, and frequency of paroxysmal episodes than in the p.Asp801Asn mutation group has been recorded, although no statistically significant differences have been achieved. Moreover, even if the difference did not reach a significant value, a greater efficacy on reducing intensity and duration of episodes was observed in this group than in p.Glu815Lys patients. These results are not easily interpretable, given the numerous variables involved in response to treatment. Future studies may further clarify the relationship between genotype and treatment response. Understanding the molecular pathogenesis and the neuronal circuits involved in the disease would guide the therapeutic choices. Alternating hemiplegia of childhood is a heterogeneous disorder characterized by dysfunctions involving different neuronal networks, causing paroxysmal and nonparoxysmal movement disorders, epilepsy, and cognitive deficits. Our results confirm that different ATP1A3 gene mutations are related to different phenotypic features. Due to the disease's rarity, the sample size may have limited the study's statistical power. This limitation mainly concerns the group of patients with various mutations, including p.Gly947Arg mutation, for which it was not possible to conduct separate statistical analyzes. Further studies involving a more extensive patient sample could better clarify these differences and increase knowledge about gene functions and different mutations' role. Another possible bias is related to the wide age range of the patients' cohort and to the different mean ages in which the 3 groups were studied. However, we assume that these variations did not alter the clinical data comparison, considering that literature data seem to exclude a degenerative course in AHC and a clear progression of symptoms over the years (27).

Prediction of the disease's development and severity using genotype information is the primary aim of genotypephenotype correlation studies. This knowledge is necessary to evaluate new therapeutic approaches, provide a more accurate prognosis to patients and caregivers, and develop the most appropriate care management guidelines. An increasingly in-depth knowledge of the disease in its clinical and genetic aspects will allow for more effective management and treatment.

\section{DATA AVAILABILITY STATEMENT}

Data of mutations reported within this study have been deposited in Leiden Open Variation Database (databases.lovd.nl/shared/ references/DOI:10.3389/fneur.2021.658451).

\section{I.B.AHC CONSORTIUM}

Maria Teresa Bassi, Claudio Zucca, Edvige Veneselli, Elisa De Grandis, Michela Stagnaro, Filippo Franchini, Maria Rosaria Vavassori, Melania Giannotta, Giuseppe Gobbi, Tiziana Granata, Nardo Nardocci, Francesca Ragona, Emanuela Abiusi, Agnese Novelli, Fiorella Gurrieri, Giovanni Neri, Francesco Danilo Tiziano, Federico Vigevano, Alessandro Capuano, Stefano Sartori.

\section{AUTHOR CONTRIBUTIONS}

RC, MS, and EDG: conception and design of the study. RC, MS, LP, MC, I.B.AHC Consortium, and EDG: acquisition and analysis. RC, MS, LP, MC, LN, and EDG: drafting of the manuscript or tables. All authors contributed to the article and approved the submitted version.

\section{ACKNOWLEDGMENTS}

We want to thank all families of our patients and AISEA Onlus (Italian Association of Alternating Hemiplegia of Childhood) for their unconditional support. This work was developed within the framework of the DINOGMI Department of Excellence of MIUR 2018-2022 (law 232/2016). 


\section{REFERENCES}

1. Verret S, Steele JC. Alternating hemiplegia in childhood: a report of eight patients with complicated migraine beginning in infancy. Pediatrics. (1971) 47:675-80.

2. Samanta D. Management of alternating hemiplegia of childhood: a review. Pediatr Neurol. (2020) 103:12-20. doi: 10.1016/j.pediatrneurol.2019.10.003

3. Uchitel J, Helseth A, Prange L, McLean M, Ghusayni R, Sachdev M, et al. The epileptology of alternating hemiplegia of childhood. Neurology. (2019) 93:e1248-59. doi: 10.1212/WNL.0000000000008159

4. Jasien JM, Bonner M, D'alli R, Prange L, Mclean M, Sachdev M, et al. Cognitive, adaptive, and behavioral profiles and management of alternating hemiplegia of childhood. Dev Med Child Neurol. (2019) 61:54754. doi: $10.1111 / \mathrm{dmcn} .14077$

5. Wallace K, Uchitel J, Prange L, Jasien J, Bonner M, D'Alli R, et al. Characterization of severe and extreme behavioral problems in patients with alternating hemiplegia of childhood. Pediatr Neurol. (2020) 111:512. doi: $10.1016 /$ j.pediatrneurol.2020.06.012

6. Sweney MT, Silver K, Gerard-Blanluet M, Pedespan J-M, Renault F, Arzimanoglou A, et al. Alternating hemiplegia of childhood: early characteristics and evolution of a neurodevelopmental syndrome. Pediatrics. (2009) 123:e534-41. doi: 10.1542/peds.2008-2027

7. Bourgeois M, Aicardi J, Goutières F. Alternating hemiplegia of childhood. $J$ Pediatr. (1993) 122:673-9. doi: 10.1016/S0022-3476(06)80003-X

8. Mikati MA, Kramer U, Zupanc ML, Shanahan RJ. Alternating hemiplegia of childhood: clinical manifestations and long-term outcome. Pediatr Neurol. (2000) 23:134-41. doi: 10.1016/S0887-8994(00)00157-0

9. Rosewich H, Thiele H, Ohlenbusch A, Maschke U, Altmüller J, Frommolt P, et al. Heterozygous de-novo mutations in ATP1A3 in patients with alternating hemiplegia of childhood: a whole-exome sequencing gene-identification study. Lancet Neurol. (2012) 11:764-73. doi: 10.1016/S1474-4422(12)70182-5

10. Heinzen EL, Swoboda KJ, Hitomi Y, Gurrieri F, Nicole S, de Vries B, et al. De novo mutations in ATP1A3 cause alternating hemiplegia of childhood. Nat Genet. (2012) 44:1030-4. doi: 10.1038/ng.2358

11. Heinzen EL, Arzimanoglou A, Brashear A, Clapcote SJ, Gurrieri F, Goldstein DB, et al. Distinct neurological disorders with ATP1A3 mutations. Lancet Neurol. (2014) 13:503-14. doi: 10.1016/S1474-4422(14)70011-0

12. Ishii A, Saito Y, Mitsui J, Ishiura H, Yoshimura J, Arai H, et al. Identification of ATP1A3 mutations by exome sequencing as the cause of alternating hemiplegia of childhood in Japanese patients. PLOS ONE. (2013) 8:e56120. doi: 10.1371/journal.pone.0056120

13. Panagiotakaki E, De Grandis E, Stagnaro M, Heinzen EL, Fons C, Sisodiya S, et al. Clinical profile of patients with ATP1A3 mutations in alternating hemiplegia of childhood-a study of 155 patients. Orphanet J Rare Dis. (2015) 10:123. doi: 10.1186/s13023-015-0335-5

14. Ikeda K, Satake S, Onaka T, Sugimoto H, Takeda N, Imoto K, et al. Enhanced inhibitory neurotransmission in the cerebellar cortex of Atpla3-deficient heterozygous mice. J Physiol. (2013) 591:3433-49. doi: 10.1113/jphysiol.2012.247817

15. Viollet L, Glusman G, Murphy KJ, Newcomb TM, Reyna SP, Sweney M, et al. Alternating hemiplegia of childhood: retrospective genetic study and genotype-phenotype correlations in 187 subjects from the US AHCF registry. PLoS ONE. (2015) 10:e0127045. doi: 10.1371/journal.pone.0127045

16. Yang $\mathrm{X}$, Gao $\mathrm{H}$, Zhang J, Xu X, Liu X, Wu X, et al. ATP1A3 mutations and genotype-phenotype correlation of alternating hemiplegia of childhood in Chinese patients. PLoS ONE. (2014) 9:e97274. doi: 10.1371/journal.pone.0097274

17. Demos MK, van Karnebeek CD, Ross CJ, Adam S, Shen Y, Zhan SH, et al. A novel recurrent mutation in ATP1A3 causes CAPOS syndrome. Orphanet $J$ Rare Dis. (2014) 9:15. doi: 10.1186/1750-1172-9-15
18. Paciorkowski AR, McDaniel SS, Jansen LA, Tully H, Tuttle E, Ghoneim DH, et al. Novel mutations in ATP1A3 associated with catastrophic early life epilepsy, episodic prolonged apnea, and postnatal microcephaly. Epilepsia. (2015) 56:422-30. doi: 10.1111/epi.12914

19. Sabouraud P, Riquet A, Spitz M-A, Deiva K, Nevsimalova S, Mignot C, et al. Relapsing encephalopathy with cerebellar ataxia are caused by variants involving p.Arg756 in ATP1A3. Eur J Paediatr Neurol. (2019) 23:44855. doi: 10.1016/j.ejpn.2019.02.004

20. Zagaglia S, Steel D, Krithika S, Hernandez-Hernandez L, Custodio HM, Gorman KM, et al. RHOBTB2 mutations expand the phenotypic spectrum of alternating hemiplegia of childhood. Neurology. (2021) 96:e153950. doi: 10.1212/WNL.0000000000011543

21. Bassi MT, Bresolin N, Tonelli A, Nazos K, Crippa F, Baschirotto C, et al. A novel mutation in the ATP1A2 gene causes alternating hemiplegia of childhood. J Med Genet. (2004) 41:621-8. doi: 10.1136/jmg.2003.017863

22. de Vries B, Stam AH, Beker F, van den Maagdenberg AMJM, Vanmolkot KRJ, Laan L, et al. CACNA1A mutation linking hemiplegic migraine and alternating hemiplegia of childhood. Cephalalgia Int J Headache. (2008) 28:887-91. doi: 10.1111/j.1468-2982.2008.01596.x

23. Jen JC, Wan J, Palos TP, Howard BD, Baloh RW. Mutation in the glutamate transporter EAAT1 causes episodic ataxia, hemiplegia, and seizures. Neurology. (2005) 65:529-34. doi: 10.1212/01.WNL.0000172638.58172.5a

24. Rotstein M, Doran J, Yang H, Ullner PM, Engelstad K, De Vivo DC. Glut1 deficiency and alternating hemiplegia of childhood. Neurology. (2009) 73:2042-4. doi: 10.1212/WNL.0b013e3181c55ebf

25. Capuano A, Garone G, Tiralongo G, Graziola F. Alternating hemiplegia of childhood: understanding the genotype-phenotype relationship of ATP1A3 variations. Appl Clin Genet. (2020) 13:71-81. doi: 10.2147/TACG.S210325

26. Pisciotta L, Gherzi M, Stagnaro M, Calevo MG, Giannotta M, Vavassori MR, et al. Alternating hemiplegia of childhood: pharmacological treatment of 30 Italian patients. Brain Dev. (2017) 39:521-8. doi: 10.1016/j.braindev.2017.02.001

27. Panagiotakaki E, Gobbi G, Neville B, Ebinger F, Campistol J, Nevsímalová $\mathrm{S}$, et al. Evidence of a non-progressive course of alternating hemiplegia of childhood: study of a large cohort of children and adults. Brain J Neurol. (2010) 133:3598-610. doi: 10.1093/brain/awq295

28. Richards S, Aziz N, Bale S, Bick D, Das S, Gastier-Foster J, et al. Standards and guidelines for the interpretation of sequence variants: a joint consensus recommendation of the American College of Medical Genetics and Genomics and the Association for Molecular Pathology. Genet Med. (2015) 17:40524. doi: 10.1038/gim.2015.30

29. Helseth AR, Hunanyan AS, Adil S, Linabarger M, Sachdev M, Abdelnour E, et al. Novel E815K knock-in mouse model of alternating hemiplegia of childhood. Neurobiol Dis. (2018) 119:100-12. doi: 10.1016/j.nbd.2018.07.028

30. Panagiotakaki E, Doummar D, Nogue E, Nagot N, Lesca G, Riant F, et al. Movement disorders in patients with alternating hemiplegia: "Soft" and "stiff" at the same time. Neurology. (2020) 94:e1378-e1385. doi: 10.1212/WNL.0000000000009175

Conflict of Interest: The authors declare that the research was conducted in the absence of any commercial or financial relationships that could be construed as a potential conflict of interest.

Copyright (c) 2021 Cordani, Stagnaro, Pisciotta, Tiziano, Calevo, Nobili, I.B.AHC Consortium and De Grandis. This is an open-access article distributed under the terms of the Creative Commons Attribution License (CC BY). The use, distribution or reproduction in other forums is permitted, provided the original author(s) and the copyright owner(s) are credited and that the original publication in this journal is cited, in accordance with accepted academic practice. No use, distribution or reproduction is permitted which does not comply with these terms. 